\title{
Transport Properties of Nanoporous, Chemically Forced Biological Lattices
}

Po-Nan Li, Jonathan Herrmann, Soichi Wakatsuki, Henry van den Bedem

Submitted date: 17/06/2019 - Posted date: 18/06/2019

Licence: CC BY-NC-ND 4.0

Citation information: Li, Po-Nan; Herrmann, Jonathan; Wakatsuki, Soichi; van den Bedem, Henry (2019):

Transport Properties of Nanoporous, Chemically Forced Biological Lattices. ChemRxiv. Preprint.

We numerically examine the electrochemical properties of negatively charged, nanoporous S-layers under asymmetrically forced ion transport governed by Michaelis-Menten kinetics at ultra-low concentrations.

File list (2)

Transport_Properties_Of_nanoporous_Chemically-Forced... (4.19 MiB) view on ChemRxiv • download file SI.pdf $(633.93 \mathrm{KiB})$ view on ChemRxiv • download file 


\title{
Transport properties of nanoporous, chemically forced biological lattices.
}

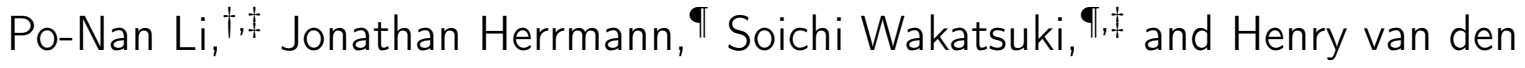 \\ Bedem $*, \ddagger, \S$ \\ $\dagger$ Department of Electrical Engineering, Stanford University, 318 Campus Dr, Stanford, CA \\ 94305 \\ $\ddagger$ Biosciences Division, SLAC National Accelerator Laboratory, Stanford University, 2575 \\ Sand Hill Rd, Menlo Park, CA 94025 \\ \Department of Structural Biology, Stanford University, 318 Campus Dr, Stanford, CA \\ 94305 \\ $\S$ Department of Bioengineering and Therapeutic Sciences, University of California San \\ Francisco, 1700 4th St, San Francisco, CA 94158 \\ E-mail: vdbedem@stanford.edu
}

\begin{abstract}
Permselective nanochannels are ubiquitous in biological systems, controlling ion transport and maintaining a potential difference across a cell surface. Surface layers (S-layers) are proteinaceous, generally charged lattices punctuated with nano-scale pores that form the outermost cell envelope component of virtually all archaea and many bacteria. Ammonia Oxidizing Archaea (AOA) obtain their energy exclusively from oxidizing ammonia directly below the S-layer lattice, but how the charged surfaces and nanochannels affects availability of $\mathrm{NH}_{4}^{+}$at the reaction site is unknown. Here, we examine the electrochemical properties of negatively charged S-layers for asymmetrically forced ion transport governed by MichaelisMenten kinetics at ultra-low concentrations. Our 3-dimensional electro-diffusion reaction simulations revealed that a negatively charged S-layer can invert the potential across the nanochannel to favor chemically forced $\mathrm{NH}_{4}^{+}$transport, analogous to polarity switching in nanofluidic field effect transistors. Polarity switching was not observed when only the interior of the nanochannels was charged. We found that S-layer charge, nanochannel geometry and enzymatic turnover rate are finely tuned to elevate $\mathrm{NH}_{4}^{+}$concentration at the active site, potentially enabling AOA to occupy nutrient-poor ecological niches. Strikingly, and in contrast to voltage-biased systems, magnitudes of the co- and counter-ion currents in the charged nanochannels were nearly equal and amplified disproportionally to the $\mathrm{NH}_{4}^{+}$current. Our simulations suggest that engineered arrays of crystalline proteinaceous membranes could find unique applications in industrial energy conversion or separation processes.
\end{abstract}

keywords nanochannels, chemically forced transport, surface layers, proteinaceous membrane

Virtually all living cells control electrolyte concentrations within their volume by selectively exchanging charged ion species with the environment through nano-scale ion channels and pumps. ${ }^{\text {[1] }}$ These macromolecular nanochan- nels enforce biochemical selectivity, can control ion transport direction across the lipid membrane and respond to external stimuli.

Engineering these signature properties of biological nanochannels into synthetic, nanofluidic 

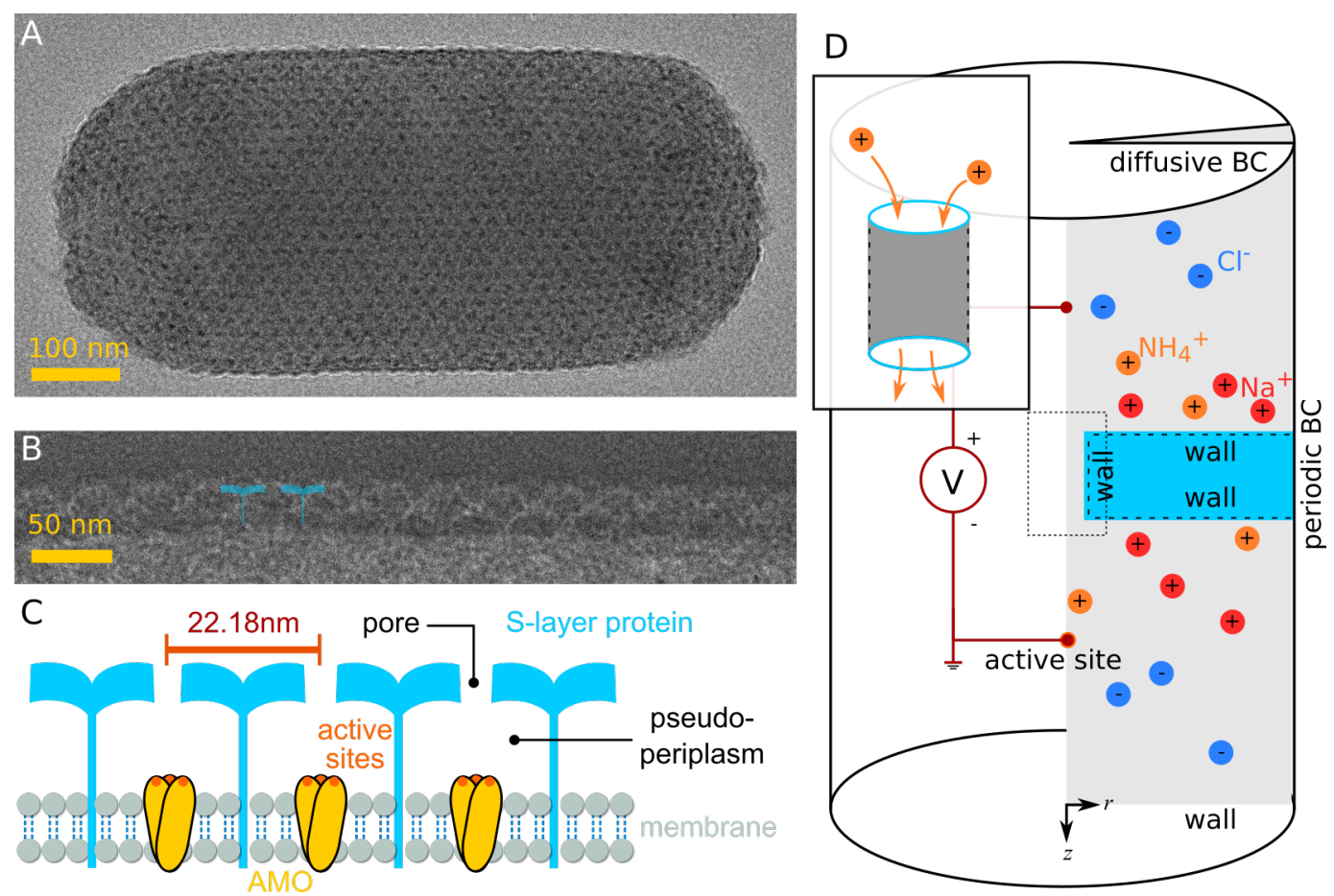

Figure 1: (A) Heavy-metal stained (1\% uranyl acetate) transmission electron micrograph of an Candidatus Nitrosoarchaeum limnia strain SFB1 cell and its S-layer. (B) A side view of the AOA S-layer stained with $2 \%$ ammonium molybdate illustrates the geometry of S-layer proteins (SLPs). (C) Schematic of the molecular organization of the AOA cell-surface. SLPs are shown in blue. The membrane (grey) associated AMO trimer is shown in yellow, with the AMO active sites in orange. (D) Schematic of the cylindrical simulation domain together with the three ion species. An S-layer (blue) with a pore is placed in the center of the domain. Negative charges are uniformly distributed on the surface of the S-layer and in the nanochannel. A reaction site (ion sink) is placed at the center axis of the simulation domain, below the nanochannel. The potential difference across the nanochannel is measured between the active site, and a site equidistant from the S-layer in extracellular space. Shaded wedge indicates the actual simulation domain, after taking domain symmetries into account. Inset: a nanochannel of $1.3 \mathrm{~nm}$ diameter connects the upper reservoir and the pseudo-perplasmic space (PPS).

devices can have important industrial appli-

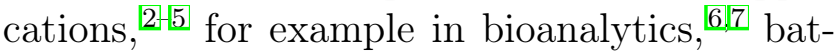
tery design, $\stackrel{89}{80}$ or harvesting energy from salinity gradients. 10 Transport properties of synthetic nanochannels are typically studied under electrical forcing, by an externally applied bias potential. 1112 In those scenarios, charged nanochannels can give rise to unusual non-linear electrokinetic effects, for example diode-like current rectification, current limiting or overlimiting behavior.13 However, despite the promise of nanofluidic devices, interactions of solutes with charged surfaces at the nanoscale remain poorly understood, both theoretically and experimentally. ${ }^{16}$ In particular, characterizing electrokinetic behavior in response to coupling to other driving forces, e.g., pressure-driven transport ${ }^{17}$ remains largely unexplored.
In biological systems, an enzymatic reaction which converts its substrate into product in nano-porous confinement is an example of chemically forced transport. Dissipation of concentration and charge by an ion sink acting on a solute induces an electro-chemical potential, forcing ions across the nano-channel. Ammonia Oxidizing Archaea (AOA) 1920 are are among the most abundant and ubiquitous organisms in the biosphere, ${ }^{2122}$ and play a major role in global nitrogen cycling. AOA are chemolithoautotrophs; their metabolism depends exclusively on ammonia oxidation by an ammonia monooxygenase (AMO) metalloenzyme complex and a copper-based electron transport system.1923 AOA have an extraordinarily high affinity for their substrate, with cultures of Nitrosopumilus maritimus strain SCM1 showing exponential growth at substrate con- 
centrations (measured as $\mathrm{NH}_{3}+\mathrm{NH}_{4}^{+}$) below $10 \mathrm{nM}$. The precise cellular location of the archaeal AMO active site is unknown, but the bacterial homologue indicates a location within the pseudo-periplasmic space (PPS), above the inner membrane and underneath a protein coat known as the surface layer (S-layer) ${ }^{2324}$ (Figure $1(\mathrm{~A}-\mathrm{C}))$.

S-layers are crystalline lattices punctuated with nanoscale pores that form the outermost cell envelope component of virtually all archaea and many bacteria. ${ }^{24}$ Although functionally diverse, the S-layer structure acts as a particle sieve to protect the cell. ${ }^{27 / 28}$ Nano-scale lattices affect the diffusion behavior of lowconcentration solutes, even if they are significantly smaller than the pore size. ${ }^{[29}$ S-layer proteins are highly charged, and it is believed that a charged S-layer surface mitigates diffusion limitations imposed by a physical permeability barrier. 1830 We previously established that S-layer charges can elevate $\mathrm{NH}_{4}^{+}$concentration throughout PPS, using a 2-D electro-diffusion reaction simulation and a linear approximation to the Michaelis-Menten kinetics. .30$]$

Here, we focus on transport characteristics of the S-layer nanochannels in response to an electro-chemical potential applied by an ion sink acting on a low-concentration cationic electrolyte in solution. Importantly, although the ionic solution is composed of three ion species, $\mathrm{Na}^{+}, \mathrm{Cl}^{-}$, and $\mathrm{NH}_{4}^{+}$, the sink acts asymmetrically on the electrolytes; only one of the charged species, $\mathrm{NH}_{4}^{+}$, is reacted away. To distinguish such a system from traditional nanochannels under external voltage bias which affects all ion species, we will refer to it as an 'asymmetric, chemically forced nanochannel'. To the best of our knowledge, chemically forced nanochannels at steady state have not been studied in the literature before.

To determine the molecular organization of the cellular envelope of AOA for our simulations, we obtained transmission electron micrographs of Candidatus Nitrosoarchaeum

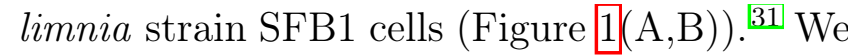
quantitatively modeled the cellular envelope of AOA with a 3-dimensional Poisson-NernstPlanck (PNP) electro-diffusion reaction trans- port model to probe how the environment, a nanoporous S-layer, AMO, its substrate, and co- and counter-ions interact to modulate electrolyte concentrations and transport properties (Figure 1(C,D), Methods).

Our system shares characteristics with a nanofluidic ion field-effect transistor (IFET). $\stackrel{3233}{3}$ The S-layer surface charges govern nanochannel ionic selectivity and conductance, analogous to an IFET's gate electrode with potential $V_{g}$. The catalytic turnover rate of the ion sink modulates the 'source-to-drain' potential $V_{d s}$. Our non-equilibrium simulations suggest that a charged S-layer elevates $\mathrm{NH}_{4}^{+}$concentration at a theoretical pseudo-periplasmic active site at low bulk concentrations. Remarkably, and in contrast to a nanochannel in an external electrical field, the electro-chemical potentials are dominated by the S-layer charges leading to electrolyte gradients perpendicular to the surfaces. The S-layer charge density, nanochannel dimensions and enzymatic rate are finely tuned to elevate substrate availability at the active site at low bulk concentrations. Near neutral S-layer surface charge densities lead to an $\mathrm{NH}_{4}^{+}$diffusion barrier in the nanochannel. However, at sufficiently negative charge densities, chemical forcing of the electrolyte generates a favorable potential difference across the nanochannel.

These simulations can help us understand how the molecular organization of the AOA cell envelope contributes to concentrating the microbe's sole energy source at the ammoniaoxidation site of the cell, enabling them to occupy ecological niches. Spatially resolved images of the microbial components together with mesoscale molecular dynamics simulations can provide important additional details on specific molecular interactions, towards a structural systems biology understanding of these ubiquitous and important organisms, and eventual rational design and bioengineering synthetic systems. 


\section{Results and Discussion}

We imaged Nitrosoarchaeum limnia cells ${ }^{31}$ stained with either $1 \%$ uranyl acetate or $2 \%$ ammonium molybdate using transmission electron microscopy (Figure 1(A,B)). ${ }^{34}$ The images revealed a crystalline outer cell envelope of S-layer proteins (SLPs) organized in hexagonal patterns, characteristic of similar archaeal

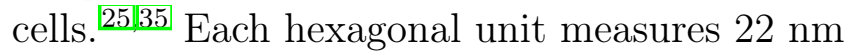
in diameter, and connects extracellular space to PPS with nano-scale channels Figure 1(C)). The distance from the membrane to the center of the S-layer is 20nm (Figure 1(D)). Thermodynamic characterization of the S-layer of Nitrosopumilus maritimus ${ }^{118}$ and the measured zeta potential of $N$. Limnia $a^{\sqrt{30}}$ revealed that cellular envelopes of AOA are highly charged. Ion exchange between extracellular space and PPS takes place through the charged nano-channel.

We capitalized on the symmetries of the system to formulate a pseudo three-dimensional simulation. We approximate the hexagonal cell

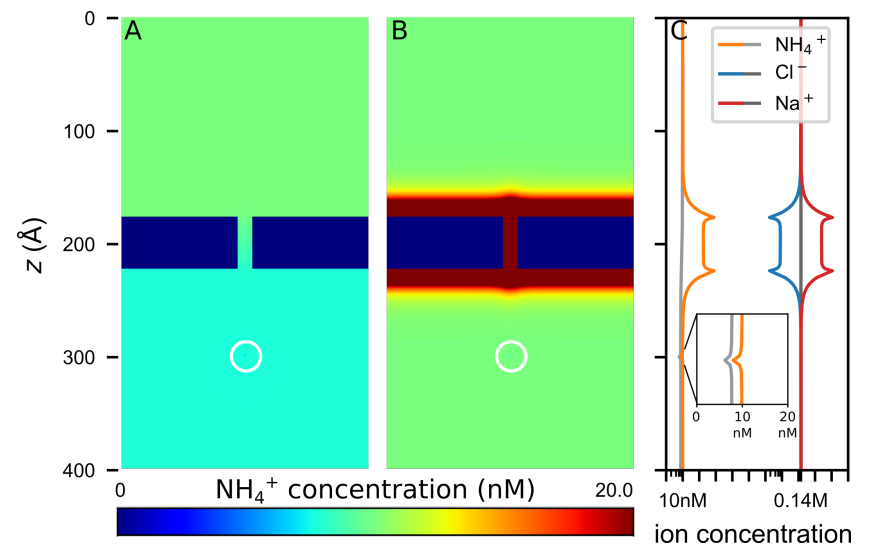

Figure 2: Concentration profile of $\mathrm{NH}_{4}^{+}$at steady state (A) with a neutral S-layer, (B) with a negatively charged S-layer. Surface charges on the S-layer lead to the formation of an EDL ((B), dark red) and create a concentration gradient near the surface layer. Note that the EDLs in the nano-channel overlap. The white circle marks the location of the ion sink. Bulk concentrations equal $c_{i=\mathrm{NH}_{4}^{+}}^{\text {bulk }}=10 \mathrm{nM}, c_{i=\mathrm{Cl}^{-}}^{\text {bulk }}=0.14$ $\mathrm{M}$, and $c_{i=\mathrm{Na}^{+}}^{\text {bulk }}=\left(0.14-10^{-8}\right) \mathrm{M}$. The S-layer surface charge density is $\rho=0.02 \mathrm{e}^{-} \AA^{-2}$. (C) $\mathrm{NH}_{4}^{+}, \mathrm{Cl}^{-}$ and $\mathrm{Na}^{+}$ion concentrations across the nanochannel. Gray: with a neutral S-layer; orange, blue, red: with a negatively charged S-layer. with a cylindrical simulation domain $11 \mathrm{~nm}$ in radius and $40 \mathrm{~nm}$ in height (Figure 1(D)). A $4.5 \mathrm{~nm}$ thick, impenetrable S-layer is located at the center $(z=20 \mathrm{~nm})$ of the simulation domain, with a nanochannel centered at the $z$-axis. The diameter of the nanochannel is set to $1.3 \mathrm{~nm}, \frac{30}{3}$ unless stated otherwise. The bulk ion concentrations $c_{i}^{\text {bulk }}$ in our simulations match those of the marine habitat of AOA (Table 2): $c_{i=\mathrm{NH}_{4}^{+}}^{\text {bulk }}=10 \mathrm{nM}, c_{i=\mathrm{Cl}^{-}}^{\text {bulk }}=0.14 \mathrm{M}$, and $c_{i=\mathrm{Na}^{+}}^{\mathrm{bulk}}=\left(0.14-10^{-8}\right) \mathrm{M}$ to maintain bulk electro-neutrality. The S-layer surface charge density equals $\rho=0.02 \mathrm{e}^{-} \AA^{-2}$.

\section{S-layer surface charge elevates steady-state $\mathrm{NH}_{4}^{+}$concentrations in pseudo-periplasmic space}

The S-layer surface charges lead to the formation of an electric double layer (EDL), which extends approximately $0.8 \mathrm{~nm}$ on both sides of the S-layer (Figure 2(A,B)).

At steady state, $\mathrm{NH}_{4}^{+}$and $\mathrm{Na}^{+}$concentrations were elevated by an order of magnitude near the S-layer, while the $\mathrm{Cl}^{-}$concentration is similarly reduced compared to bulk (Figure 2(C)). Consequently, at the boundaries of the EDL, above and below the S-layer, we observe a strong ion concentration gradient (Figure 2(C)). In the nanochannel, cation concentrations were enriched compared to the neutral channel $\left(c_{i=\mathrm{Na}^{+}}^{\mathrm{bulk}}=2.46 \mathrm{M}\right.$ vs. $\left.0.14 \mathrm{M}\right)$, while the anion concentration was sharply reduced $\left(c_{i=\mathrm{Cl}^{-}}^{\text {bulk }}=0.008 \mathrm{M}\right.$ vs. $\left.0.14 \mathrm{M}\right)$.

The active site itself is positioned too far from the S-layer to experience the sharp concentration gains of the EDL (Figure 2(B)). However, at steady state the $\mathrm{NH}_{4}^{+}$concentration in pseudo-periplasmic space was elevated nearly uniformly by $29.4 \%$ for the charged Slayer compared to the neutral S-layer, in reasonable agreement with our previous 2-D simulation and linear kinetics. 30 


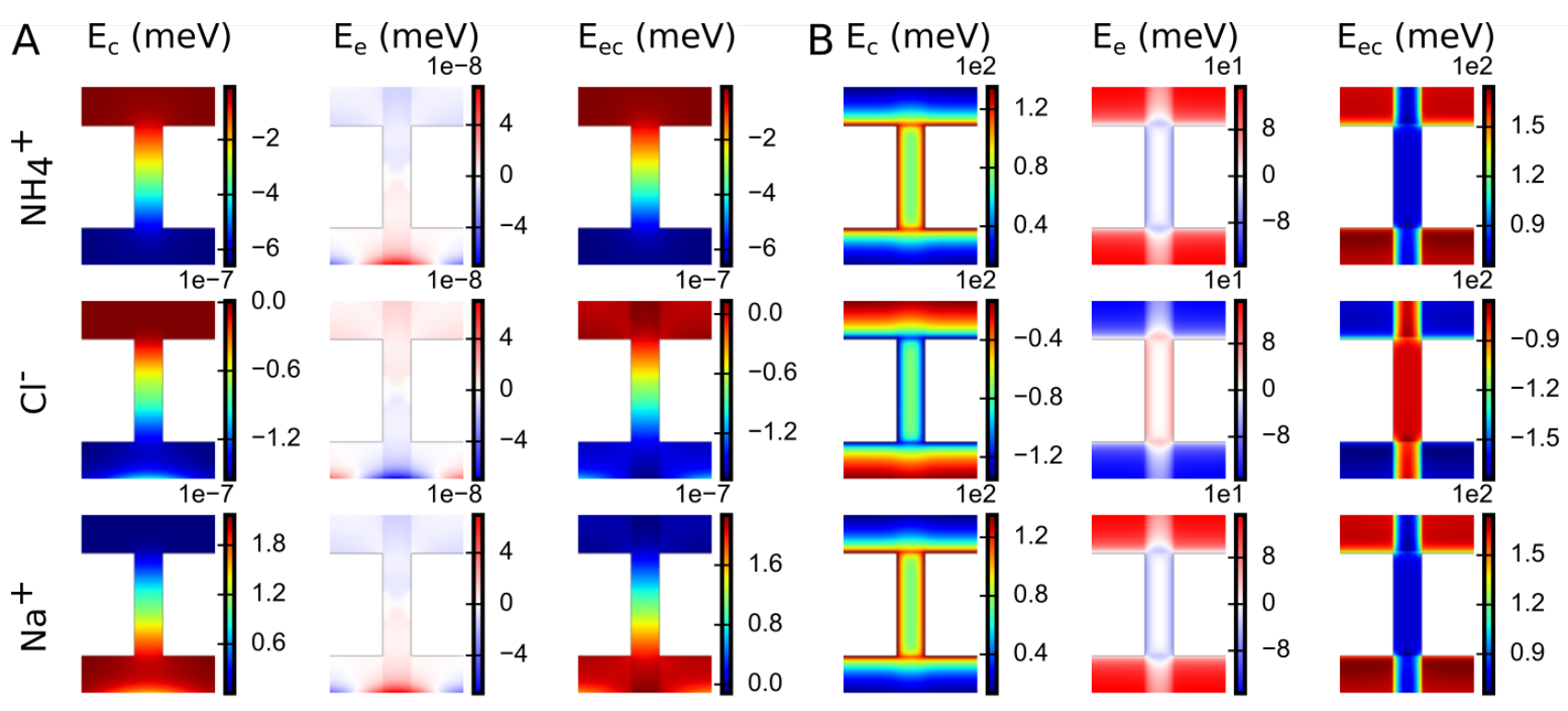

Figure 3: Steady state electro-chemical potential for $(A)$ neutral and $(B)$ charged nanochannel. In the neutral case, the electro-chemical potential of $\mathrm{NH}_{4}^{+}$and co- and counter-ions shows regularly spaced equipotential surfaces, perpendicular to the pore walls. In the charged case, the electrical and chemical potentials are parallel to the pore walls, in stark contrast to a nanochannel under external voltage bias.

Electro-chemical potentials across the nanochannel

At steady state in the neutral case, the direction of the $\mathrm{NH}_{4}^{+}$electro-chemical gradient $\nabla E_{e c, \mathrm{NH}_{4}^{+}}$ across the pore was towards PPS (Figure 3). The pore was mildly resistive for $\mathrm{NH}_{4}^{+}$, indicated by an electrical potential that was $31.5 \mu \mathrm{V}$ higher in PPS than in extracellular space, i.e., a weak electric field $\mathbf{E}$ was oriented outward through the nanochannel (Methods). $E_{e c, \mathrm{NH}_{4}^{+}}$ was dominated by its chemical component, which was eight orders of magnitude larger than the electrical component. $\mathrm{Na}^{+}$and $\mathrm{Cl}^{-}$presumably responded to the $\mathrm{NH}_{4}^{+}$-driven charge redistribution, indicated by chemical potentials that compensated for $\mathrm{NH}_{4}^{+}$concentrations. For the $\mathrm{Na}^{+}$and $\mathrm{Cl}^{-}$ion species, the electro-chemical potential was eight orders of magnitude smaller than $E_{e c, \mathrm{NH}_{4}^{+}}$. Their chemical potential was of the same order as the electrical potential, reflecting the difference in bulk concentration between $\mathrm{NH}_{4}^{+}$and the co- and counter-ion. $\mathrm{Cl}^{-}$ experienced a marginally conductive nanochannel, while $\mathrm{Na}^{+}$was marginally resistive. The electrical potentials in the nanochannel were highly symmetric across the three ion species.

In the charged case, the system entered a dramatically different regime. Strikingly, the polarity of the nanochannel inverted with a charged $\left(\rho=0.02 \mathrm{e}^{-\AA^{-2}}\right.$ ) S-layer. The electrical potential was $95.2 \mu \mathrm{V}$ higher in extracellular space than in the PPS: $\mathbf{E}$ was oriented inward. Unlike the neutral case, or the case with an externally applied voltage bias acting symmetrically on ion species, ${ }^{44}$ electro-chemical equipotential surfaces were no longer perpendicular to the nano-pore surface (Figure 3(B)). Instead, electric and chemical components of the ion potentials were dominated by the surface charges leading to electro-chemical potentials parallel to the nano-pore surfaces. The electrical and chemical components of the potentials were on the same order of magnitude for all ion species. In the neutral case $E_{e c, \mathrm{Na}^{+}}$and $E_{e c, \mathrm{Cl}^{-}}$responded to $\mathrm{NH}_{4}^{+}$-driven charge redistribution, but in the charged case $E_{e c, \mathrm{Na}^{+}}$was indistinguishable from $E_{e c, \mathrm{NH}_{4}^{+}}$while $E_{e c, \mathrm{Cl}^{-}}$was inverted from $E_{e c, \mathrm{NH}_{4}^{+}}$.

\section{Ion currents}

The lack of a strong inward directed electrochemical gradient across the pore (Figure $3 \mathrm{~B}$ ) raises the question how a charged S-layer modulates influx of $\mathrm{NH}_{4}^{+}$into PPS. In the neutral case, we observed a 'funnel'-like $\mathrm{NH}_{4}^{+}$ion flux near the entrance and exit of the nanochannel (Figure 4(A) top left panel). In the charged case, our simulations indicated strong $\mathrm{NH}_{4}^{+}$cur- 

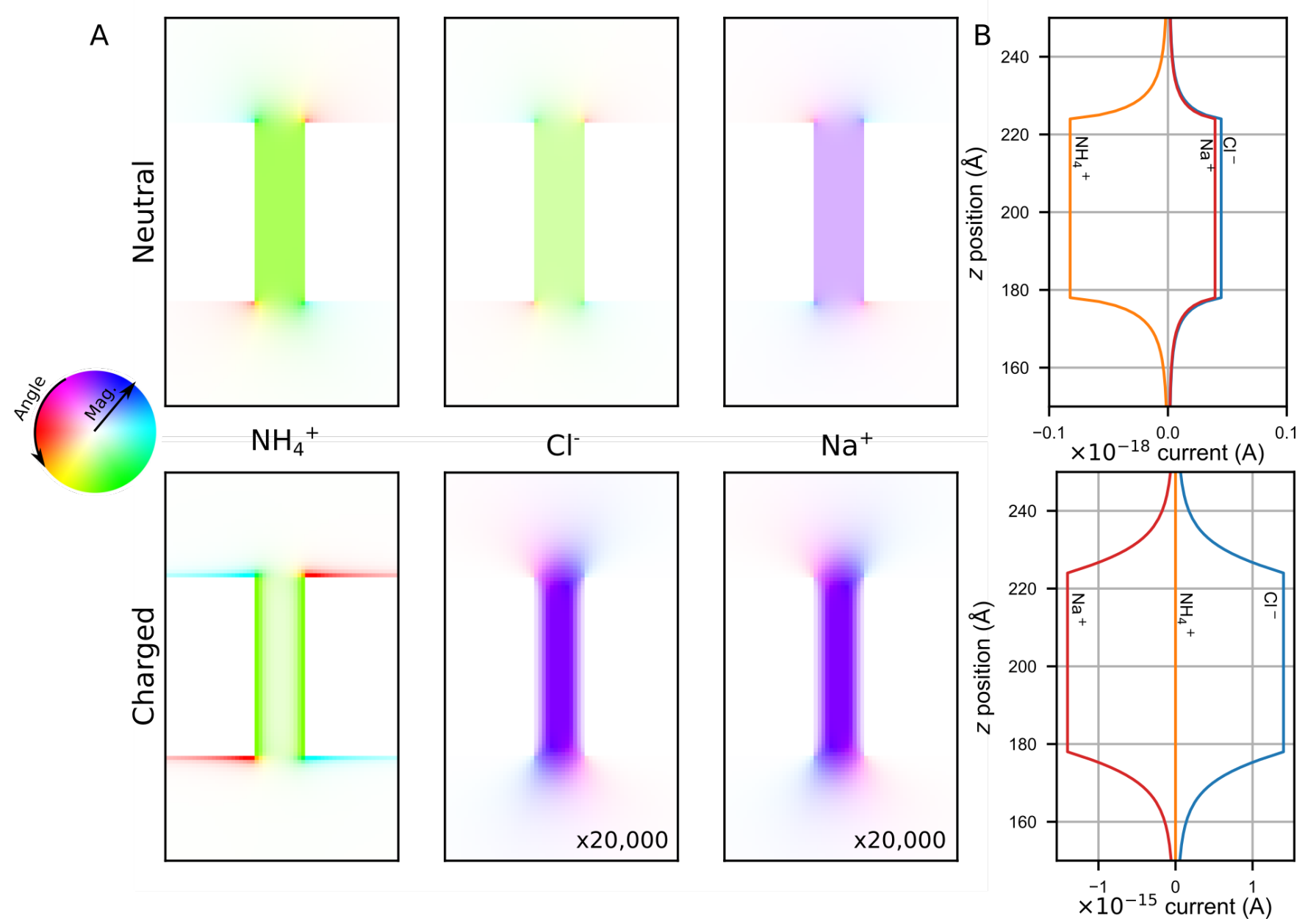

Figure 4: (A) Current magnitude and direction of the ion species with a neutral (upper panel) and charged (lower panel) S-layer with surface charge density $0.02 \mathrm{e}^{-} \AA^{-2}$. The saturation of color indicates the magnitude of the ion current and the hue indicates the direction of the ion current. Scale of magnitude in charged $\mathrm{Cl}^{-}$and $\mathrm{Na}^{+}$(lower middle and right) is 20,000 times greater than in other panels. (B) Current profiles for $\mathrm{Na}^{+}, \mathrm{Cl}^{-}$, and $\mathrm{NH}_{4}^{+}$along the central axis in a neutral (upper) and charged (lower) setting.

rents in the EDL parallel to the S-layer and, surprisingly, parallel to its electrical and chemical equipotential surfaces (Figure 4 (A) bottom left panel). $\mathrm{NH}_{4}^{+}$currents flowed towards the nanochannel entrance along the top (extracellular) surface of the S-layer, and away from the nanochannel exit along the bottom (enzyme facing) surface. In the nanochannel, $\mathrm{NH}_{4}^{+}$currents were directed also along the surface. This suggests that the charged S-layer acts as a $\mathrm{NH}_{4}^{+}$ reservoir. At the enzyme facing side of the Slayer, $\mathrm{NH}_{4}^{+}$ions diffuse out of the EDL towards the active site (Figure S2). These ions are replaced by transport along the slipping plane at the extracellular and nanochannel surface of the S-layer (Figure 4(A).

In both the neutral and charged cases co- and counter-ions revealed no significant current along the top or bottom S-layer surfaces (Figure $2 \mathrm{~A}$ ).

In the neutral case, the current carried by $\mathrm{NH}_{4}^{+}$was $\mathrm{I}_{4}^{+}=8.31 \times 10^{-20}$ A (Figure $4(\mathrm{~B})$ top panel; Table 11). Co- and counter-ions had near equal current magnitude Figure 4(B) top panel), jointly compensating for $\mathrm{I}_{\mathrm{NH}_{4}^{+}}$: $\mathrm{I}_{\mathrm{Na}^{+}+\mathrm{Cl}^{-}}=-8.31 \times 10^{-20} \mathrm{~A}$. In the charged case, the $\mathrm{NH}_{4}^{+}$current increased by $28 \%$ to $\mathrm{I}_{\mathrm{NH}_{4}^{+}}=1.06 \times 10^{-19}$ A Figure 4(B) top panel). Strikingly, while $\mathrm{Na}^{+}$and $\mathrm{Cl}^{-}$currents still neutralized the total current $\left(\mathrm{I}_{\mathrm{Na}^{+}+\mathrm{Cl}^{-}}=-1.06 \times\right.$ $10^{-19} \mathrm{~A}$ ), each was amplified by four orders of magnitude compared to the neutral case (Table 11. The current of $\mathrm{Cl}^{-}$had reversed sign. Even more surprising, co- and counter-ion cur- 
Table 1: Current, potential and conductance data in a neutral and charged nanochannel with surface charge density $0.02 \mathrm{e}^{-} / \AA^{2}$. The potential is measured as depicted in Figure 1(B)

\begin{tabular}{rrrr}
\multicolumn{5}{c}{ Neutral } \\
\hline \hline Species & $\mathrm{NH}_{4}^{+}$ & $\mathrm{Cl}^{-}$ & $\mathrm{Na}^{+}$ \\
\hline Current & $-8.31 \mathrm{E}-20 \mathrm{~A}$ & $4.41 \mathrm{E}-20 \mathrm{~A}$ & $3.89 \mathrm{E}-20 \mathrm{~A}$ \\
Potential & $-3.15 \mathrm{E}-05 \mathrm{~V}$ & & \\
Conductance & $-2.63 \mathrm{E}-15 \mathrm{~S}$ & $1.40 \mathrm{E}-15 \mathrm{~S}$ & $1.23 \mathrm{E}-15 \mathrm{~S}$ \\
\hline \multicolumn{4}{c}{ Charged } \\
\hline Species & $\mathrm{NH}_{4}^{+}$ & $\mathrm{Cl}^{-}$ & $\mathrm{Na}^{+}$ \\
\hline \hline Current & $1.06 \mathrm{E}-19 \mathrm{~A}$ & $1.40 \mathrm{E}-15 \mathrm{~A}$ & $-1.40 \mathrm{E}-15 \mathrm{~A}$ \\
Potential & $9.52 \mathrm{E}-05 \mathrm{~V}$ & & \\
Conductance & $1.11 \mathrm{E}-15 \mathrm{~S}$ & $1.47 \mathrm{E}-11 \mathrm{~S}$ & $-1.47 \mathrm{E}-11 \mathrm{~S}$ \\
\hline
\end{tabular}

rents did not reflect cation selectivity despite a concentration difference between $\mathrm{Na}^{+}$and $\mathrm{Cl}^{-}$in the nanochannel exceeding two orders of magnitude (Figure 2B). Instead, co- and counter-ion current magnitudes were near equal ((Figure 4(B) bottom panel). By contrast, in external-voltage biased nanochannels with negative surface charges, anionic $\left(\mathrm{Cl}^{-}\right)$currents are reduced with respect to $\mathrm{Na}^{+}$owing to lower concentrations in the nanochannel. We postulate that the current response in a chemically forced nanochannel is limited by the least conductive ion species. Thus, an $\mathrm{NH}_{4}^{+}$-driven potential difference solicits a current response in $\mathrm{Na}^{+}$and $\mathrm{Cl}^{-}$which is symmetric to enforce electro-neutrality but limited by the ability of the $\mathrm{Cl}^{-}$ion concentration to carry current.

The simulations indicated a sharp increase in co- and counter-ion currents with a charged Slayer compared to a neutral S-layer (Table 1). In both cases $\mathrm{I}_{\mathrm{Na}^{+}+\mathrm{Cl}^{-}}$compensated $\mathrm{I}_{\mathrm{NH}_{4}^{+}}$, but it remains unclear why $\mathrm{I}_{\mathrm{Na}^{+}}$and $\mathrm{I}_{\mathrm{Cl}^{-}}$in the charged case are amplified by four orders of magnitude compared to the neutral case. We did not detect co- and counter-ion current amplification in our $2 \mathrm{D}$ simulation. ${ }^{30}$ To examine the origin of current amplification, we approximated the 2D simulation by omitting the $J_{r, i} / r$ term from Equation 11 in our 3D implementation. Simulations with this model reduced the co- and counter-ion current magnitudes comparable to those observed with the $2 \mathrm{D}$ model (Table S1), suggesting that current amplification is due to a 'concentration-focusing' effect at the radial axis of the nanochannel in the 3D model.

\section{Catalytic turnover rate and surface charges finely tuned to maximize gain}

The low $\mathrm{NH}_{4}^{+}$bulk concentration suggests that the reaction rate will be diffusion limited. To identify a range of operational reaction rates, we determined how $\mathrm{NH}_{4}^{+}$concentrations at the active site depend on the catalytic turnover rate $k_{c}$ and the surface charge density of the S-layer (Figure 5(A)). For all surface charges, including neutral, the concentration depended non-linearly on the value of $k_{c}$. Unsurprisingly, increasing $k_{c}$ significantly lowered the steady-state concentration at the active site. For a fixed value of $k_{c}$, higher surface charge densities partially compensated for the faster $\mathrm{NH}_{4}^{+}$turnover rate, maintaining a higher concentration at the active site. However, we detected no further gain when surface charge densities exceeded $0.05 \mathrm{e}^{-} \AA^{-2}$ (Figure 5 (A,B)). The enzymatic rate reached a limiting point at $k_{c} \sim 65 s^{-1}$, when ions near the active site were depleted completely independent of surface charge. Thus, the $\mathrm{NH}_{4}^{+}$turnover rate at the active site and S-layer charges are delicately balanced, and together define a narrow operating regime. 

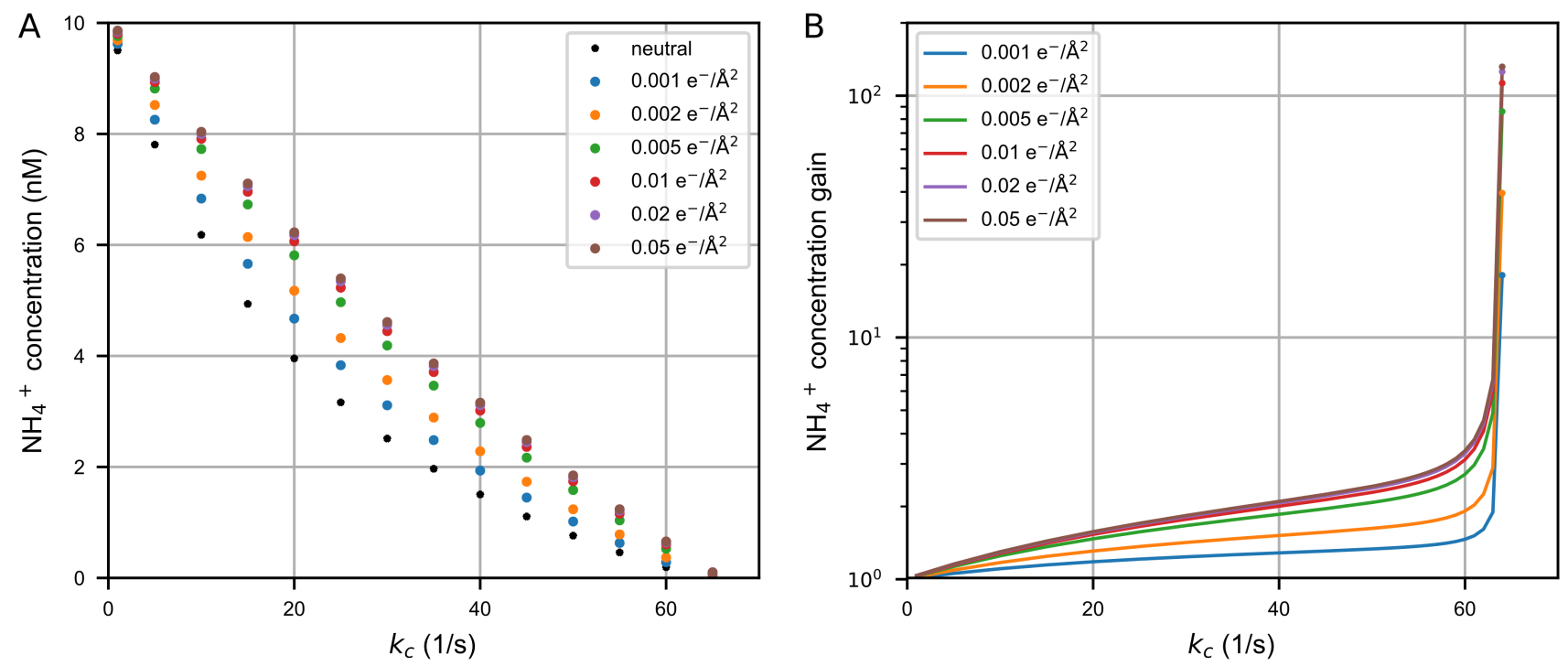

Figure 5: (A) Steady state $\mathrm{NH}_{4}^{+}$concentrations as a function of reaction constant $k_{c}$. Data points of different colors represent different surface charge density. (B) Concentration gain of $\mathrm{NH}_{4}^{+}$at the active site for different values of the S-layer surface charge. The gain sharply increased near $k_{c} \sim 65 \mathrm{~s}^{-1}$, where $\mathrm{NH}_{4}^{+}$at the active site was depleted with a neutral S-layer, but maintained a finite concentration with a charged S-layer.

\section{Current-voltage characteristics of a chemically forced nanochannel}

To determine the current-voltage characteristics of a chemically forced system, we measured the potential difference across the S-layer and the ion current in the nanochannel over a range of catalytic turnover rates $k_{c}$ and surface charge densities.

We detected a strong non-linear currentvoltage response to variations in surface charge densities (Figure 6A). At fixed turnover rates, we observed a shift towards more negative potential differences across the channel and a concomitant gain in current as the surface charge densities increased from neutral up to $\sim 0.005 \mathrm{e}^{-} \AA^{-2}$. In other words, $\mathrm{NH}_{4}^{+}$overcomes a larger (negative) potential difference across the nanochannel but can maintain a higher current, manifested as decreasing (more negative) cationic conductivity (Figure 6B).

After reaching a critical surface charge density between 0.002 and $0.005 \mathrm{e}^{-\AA^{-2}}$ depending on turnover rate (Figure 6 $6 \mathrm{~A}, \mathrm{~B}$; dashed yellow line at $0.005 \mathrm{e}^{-} \AA^{-2}$ ), further increasing surface charge densities reduced the potential difference that $\mathrm{NH}_{4}^{+}$needs to overcome across the nanochannel. We again observed a simultaneous gain in current, albeit generally smaller than that in the regime before the critical surface charge density. Thus, a substantially smaller adverse potential difference with a small gain in current suggests the nanochannel is turning more conductive for cations in this regime. Eventually, the potential (and conductance) across the nanochannel turned positive for all surface charge densities, except at the highest turnover rates. The marginal gain in current diminishes rapidly beyond $0.01 \mathrm{e}^{-} \AA^{-2}$ surface charge density, until no further gain is observed at $0.05 \mathrm{e}^{-\AA^{-2}}$ (cf. Figure $5(\mathrm{~A}, \mathrm{~B})$ ). We observed peak conductance at $\rho=0.05 \mathrm{e}^{-} \AA^{-2}$ and $k_{c}=30 \mathrm{~s}^{-1}$ (Figure 6 $6 \mathrm{~B}$ ). The turning point in conductance with respect to surface charge densities is reached at the cusp of the curves in Figure 64. The surface layer requires a turnover rate-dependent minimum negative surface charge density to enter a favorable cationic conductance regime (Figure 6B).

Simultaneously, we observed a shift towards unfavorable current-voltage characteristics at higher turnover rates independent of surface charge densities (Figure 6A). For example, at a rate of $10 \mathrm{~s}^{-1}$, the potential across the nanochannel turned positive, i.e. favorable for cations, for surface charge densities between 0.005 and $0.01 \mathrm{e}^{-} \AA^{-2}$. However, for turnover 

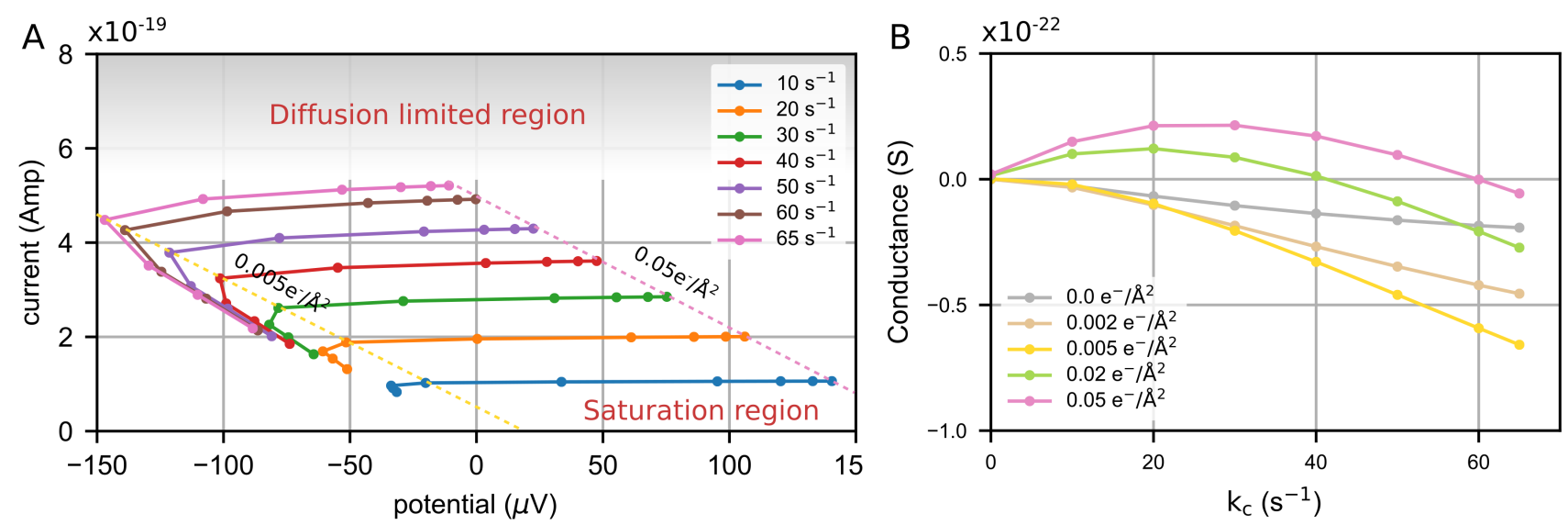

Figure 6: Current-voltage characteristics. (A) Each line corresponds to the potential difference across the S-layer and the $\mathrm{NH}_{4}^{+}$current response in the nanochannel for a fixed $k_{c}$ at different surface charge densities. From left to right, circles indicate surface charge densities at neutral, 0.001, 0.002, 0.005, 0.01, 0.02, 0.03, 0.04 and $0.05 \mathrm{e}^{-} \AA^{-2}$. The polarity across the nanochannel inverts at $0 \mathrm{~V}$. Positive values correspond to a favorable potential (conductive) across the pore for $\mathrm{NH}_{4}^{+}$. The slope of the lines is a measure of conductivity, as explained in the text. For a fixed surface charge, the reaction rate $k_{c}$ modulates the current response (dotted lines) (B) Conductance $\mathrm{G}=\operatorname{sgn}(\mathrm{V}) \mathrm{I} / \mathrm{V}$ of the data points in panel $(\mathrm{A})$ as a function of $k_{c}$, color-coded by surface charge density. Yellow and pink dotted lines in panel (A) correspond to 0.005 and $0.05 \mathrm{e}^{-} \AA^{-2}$ in panel (B).

rates $50 \mathrm{~s}^{-1}$ the system operated in a favorable potential difference only for surface charges densities $0.03 \mathrm{e}^{-} \AA^{-2}$ and higher. These results again indicate finely balanced turnover rates and surface charge densities.

These observations suggest that weak S-layer surface charge densities enrich $\mathrm{NH}_{4}^{+}$concentrations in the channel insufficiently to support chemical forcing of $\mathrm{NH}_{4}^{+}$at high turnover rates. The effect of S-layer charge is similar to the effect of the gating potential in nanofluidic field effect transistors, which controls cation depletion or enrichment. $\frac{36}{3}$ In conventional, voltage biased nanofluidic systems an increase in voltage bias will lead to ion depletion on the anodic side of the nanochannel, resulting in a non-ohmic response. ${ }^{1315137 / 38}$ By contrast, for a chemically forced nanochannel, increasing the reaction rate $k_{c}$ limits the channel conductance, leading to ion depletion at the ion sink (cathode) before current limiting behavior can be observed.

\section{Redistributed electrolyte charge densities drive concentration gain}

Next, to understand how a nanoporous S-layer strikes a balance between diffusion limitations imposed by a physical barrier and particle siev- ing, we examined the effect of nanochannel diameter on $\mathrm{NH}_{4}^{+}$concentrations at the active site and nanochannel currents.

For all bulk concentrations, we observed a non-linear dependence on the nanochannel radius (Figure 7). Unsurprisingly, the gain was highest for small diameters, but decreased rapidly for larger diameters.

The concentration gain at the active site was sharply reduced at higher $\mathrm{NH}_{4}^{+}$bulk concentrations, independent of the nanochannel diameter. The gain was highest at a $\mathrm{NH}_{4}^{+}$bulk concentration of $1 \mathrm{nM}$, and became negligibly small at bulk concentrations exceeding $1 \mu \mathrm{M}$. The small marginal gain between 10 and $1 \mathrm{nM}$ (Figure 7(A)) suggests that the maximum gain was limited by a minimum $\mathrm{NH}_{4}^{+}$concentration required at the active site to maintain the reaction. Likewise, at $10 \mu \mathrm{M}$ bulk concentration, the active site turnover rate was well below the diffusion-limited regime even with a neutral Slayer, explaining the low total gain.

The remarkable ability of S-layer charges to elevate $\mathrm{NH}_{4}^{+}$concentrations at the active site is exemplified by comparing ion currents through the nanochannel. For the smallest nanochannel diameter, we observed $\mathrm{NH}_{4}^{+}$currents up to two orders of magnitude larger with a charged S-layer compared to a neutral S-layer (Fig- 

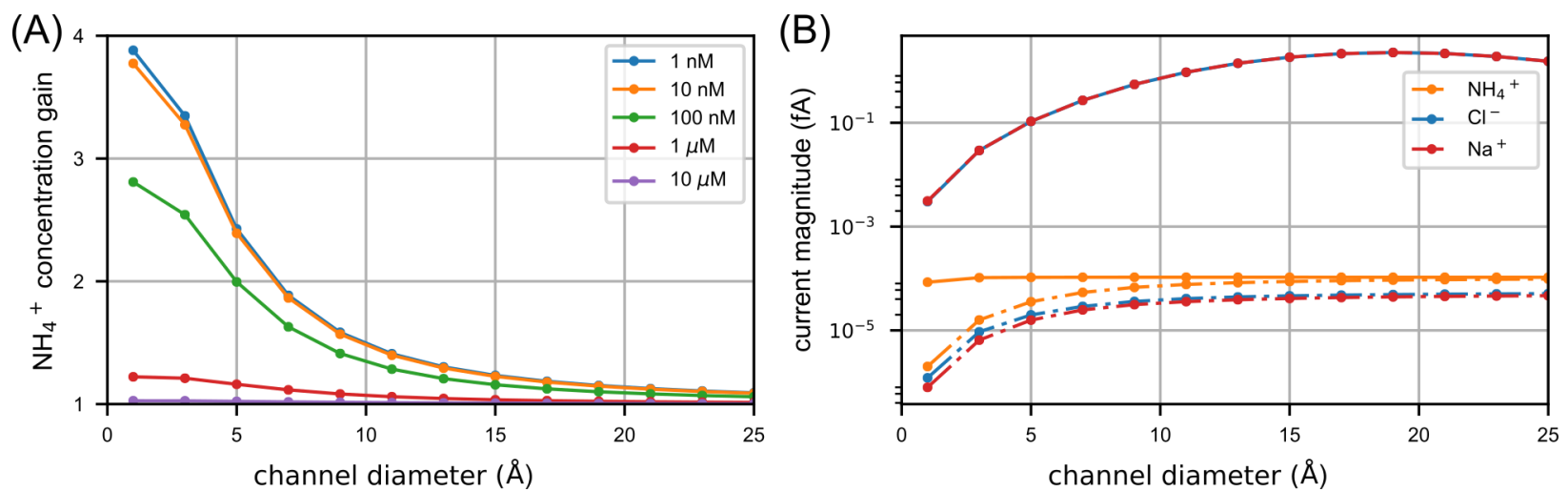

Figure 7: (A) Gain of $\mathrm{NH}_{4}^{+}$concentration at the active site for different pore diameters and bulk concentration of $\mathrm{NH}_{4}^{+}$. The gain is the ratio of concentrations between surface charge $0.02 \mathrm{e}^{-} \AA^{-2}$ and neutral. (B) Current magnitudes in the nanochannel for each ion species as a function of diameter $\left(\mathrm{NH}_{4}^{+}\right.$bulk concentration equals $10 \mathrm{nM}$ ). Solid lines: surface charge $0.02 \mathrm{e}^{-} \AA^{-2}$. Dash-dot lines: neutral S-layer.

ure 7(B)). The differences were smaller towards larger diameter. Notably, $\mathrm{NH}_{4}^{+}$currents remained in a narrow band as the nanochannel diameter increased, suggesting near perfect compensation by S-layer charges for reduced $\mathrm{NH}_{4}^{+}$ nanochannel diffusion to enable particle sieving.

The magnitude of the co- and counter-ion currents, i.e., $\mathrm{Na}^{+}$and $\mathrm{Cl}^{-}$, were amplified by four orders of magnitude (Figure 7(B)) in the case of a charged S-layer. In the neutral case, co- and counterion currents had magnitudes smaller than $\mathrm{NH}_{4}^{+}$currents. In the charged case, the co- and counterion currents were many orders of magnitude larger due to their elevated concentration and thus larger magnitude of flux. Furthermore, the co- and counter-ion currents increased by more than two orders of magnitude as the channel diameter increased from 1 to $15 \AA$. We reason that the nanochannel becomes less cation-selective, elevating the $\mathrm{Cl}^{-}$ concentration and current in the nanochannel. The $\mathrm{Na}^{+}$current reacts accordingly to neutralize the nanochannel.

To tease apart the roles of horizontal and vertical surface charges, we examined $\mathrm{NH}_{4}^{+}$fluxes in the nanochannel and active site concentrations for a neutral S-layer with a charged nanochannel and a charged S-layer with a neutral nanochannel (Figure 8(A)). Our simulations indicated that both surface only or channel only charges elevated the $\mathrm{NH}_{4}^{+}$concentrations at the active site. The contribution of surface only charges to elevating active site concentrations was stronger than that of channel only charges (Figure $8(\mathrm{~A}, \mathrm{~B})$ ). Remarkably, nanochannel charges slightly countered the effect of surface charges on the $\mathrm{NH}_{4}$ fluxes in the channel (Figure 8 $8(A, B))$. Importantly, the electric field inverted (E directed towards PPS) in the case of surface only charges, but not in the case of nanochannel only charges. These results suggest that the fixed surface charges redistribute the charge densities of the electrolyte to create a favorable electric field through the channel.

\section{Conclusion}

We analyzed transport properties of $\mathrm{NH}_{4}^{+}$ions across a nanoporous lattice under chemical forcing. The $\mathrm{NH}_{4}^{+}$ion charge and concentration dissipation at the active site does not directly act on the co- and counter-ions $\mathrm{Na}^{+}$and $\mathrm{Cl}^{-}$. Instead, $\mathrm{NH}_{4}^{+}$ion dissipation promotes a charge redistribution in the simulation domain, which, in turn, solicits a redistribution of the co- and counter-ions $\mathrm{Na}^{+}$and $\mathrm{Cl}^{-}$. We observed a starkly different transport regime with a charged S-layer compared to a neutral S-layer or compared to a voltage biased nanochannel. The charged S-layer and nanochannel showed electrochemical characteristics similar to those of a nanofluidic ion field effect transistor. Our simulations revealed that the negative charges on the S-layer can invert the polarity across 

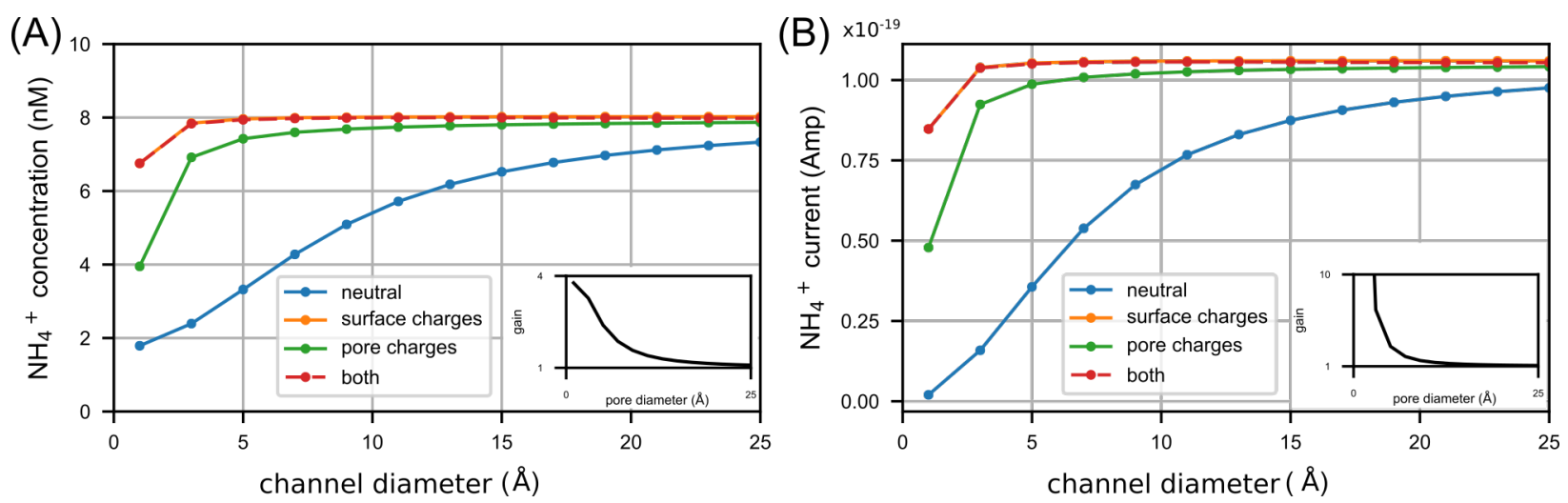

Figure 8: (A) $\mathrm{NH}_{4}^{+}$concentration at the active site with different setting of nanochannel diameter and charge configuration. Inset: gain, defined as active site $\mathrm{NH}_{4}^{+}$concentration of the charged case over the neutral case, as a function of pore diameter. (B) $\mathrm{NH}_{4}^{+}$flux across the nanochannel with different diameter and charge configurations. Inset: percentage gain, defined as the flux in the charged case over the neutral case, as a function of channel diameter.

the S-layer to favor $\mathrm{NH}_{4}^{+}$transport, amplify the counter-ions $\mathrm{Na}^{+}$and $\mathrm{Cl}^{-}$currents by several orders of magnitude, and significantly elevate the $\mathrm{NH}_{4}^{+}$concentration at an underlying enzyme active site to up to 7 -fold compared with a neutral one.

Higher enzymatic turnover rates required stronger negative surface charges to enter the regime with potential favorable for $\mathrm{NH}_{4}^{+}$. While the current carried by $\mathrm{NH}_{4}^{+}$increased concomitantly, ultimately turnover at the ion sink was diffusion limited at $k_{c} \sim 65 s^{-1}$ independent of surface charge. At the same time, for surface charge densities we measured in the laboratory, near $0.02 \mathrm{e}^{-} \AA^{-2}$, our simulations found that conductance across the pore reached a maximum at $k_{c} \sim 20 s^{-1}$, remarkably close to the measured turnover $\left(\sim 10 \mathrm{~s}^{-1}\right)$.

Our simulations necessarily simplified the geometry of the AOA molecular envelope and its components. The role of the fluid flow field was also ignored. ${ }^{45}$ Treating ions as point masses in the PNP formalism can artefactually increase ion concentrations near highly charged surfaces. Finite element numerical simulations could account for finite ion size, $\underline{46}$ and allow more complex geometries of the simulation domain than our finite difference approach. While the precise atomic structure of the AOA S-layer and nanochannels remain unknown, those of other organisms suggest the pores are conically shaped. ${ }^{25 ! 26}$ Geometric asymmetries ${ }^{48}$ and non- uniform distribution of surface charge in the nanochannel ${ }^{144}$ can lead to distinctive electrokinetic effects like current rectification, which we did not detect in our simulations. Atomically detailed molecular dynamics simulations ${ }^{\underline{47}}$ will be required to fully reveal the mechanisms and electrokinetics of chemically forced ion transport across nanoporous bio-lattices. Such insights can help design and engineer synthetic nanotechnology.

\section{Methods}

\section{Poisson-Nernst-Planck equations}

We modeled ion transport using PoissonNernst-Planck mean-field continuum theory. Ion transport in narrow nanochannels with diameters in the low (1-5) nm range is dominated by electro-phoresis, 14 and we omitted the contribution of fluid flow. ${ }^{39}$ We replaced the Poisson equation by a displacement current equation. 1041 At time $t$, the electric field $\mathbf{E}$ at $\mathbf{r}$ is governed by

$$
\frac{\partial \mathbf{E}}{\partial t}(\mathbf{r}, t)=-\sum_{i=1}^{m} \frac{\mathrm{F} z_{i}}{\epsilon} \mathbf{J}_{i}(\mathbf{r}, t),
$$


Table 2: Definitions of constants and values

\begin{tabular}{llll} 
variable & value & variable & value \\
\hline$c_{i=\mathrm{NH}_{4}^{+}}^{\text {bulk }}$ & $10 \mathrm{nM}$ & $c_{i=\mathrm{Cl}^{-}}^{\text {bulk }}$ & $0.14 \mathrm{M}$ \\
$c_{i=\mathrm{Na}^{+}}^{\text {bulk }}$ & $0.14-10^{-8} \mathrm{M}$ & $N_{A}$ & $6 \times 10^{23}$ \\
$z_{\mathrm{NH}_{4}^{+}}$ & 1 & $\mathrm{e}$ & $1.6 \times 10^{-19}$ \\
$z_{\mathrm{Cl}^{-}}$ & -1 & $F$ & $N_{A} \mathrm{e}$ \\
$z_{\mathrm{Na}^{+}}$ & 1 & $R$ & $8.314 \mathrm{~J} / \mathrm{mole}$ \\
$D_{\mathrm{NH}_{4}^{+}}$ & $1.64 \mathrm{~m}^{2} / \mathrm{s}$ & $T$ & $300 \mathrm{~K}$ \\
$D_{\mathrm{Cl}^{-}}$ & $2.03 \mathrm{~m}^{2} / \mathrm{s}$ & $\epsilon_{\text {solvent }}$ & $80 \epsilon_{0}$ \\
$D_{\mathrm{Na}^{+}}$ & $1.33 \mathrm{~m}^{2} / \mathrm{s}$ & $\epsilon_{\mathrm{S}-\text { layer }}$ & $8 \epsilon_{0}$ \\
\hline
\end{tabular}

where index $i$ denotes the ion species. The ion flux is governed by

$\mathbf{J}_{i}(\mathbf{r}, t)=-D_{i} \nabla c_{i}(\mathbf{r}, t)+\frac{\mathrm{F}}{\mathrm{R} T} D_{i} z_{i} c_{i}(\mathbf{r}, t) \mathbf{E}(\mathbf{r}, t)$,

and finally the concentration is governed by

$$
\frac{\partial c_{i}}{\partial t}(\mathbf{r}, t)=-\nabla \cdot \mathbf{J}_{i}(\mathbf{r}, t)
$$

Equations (1) and (3) are known as the Poisson-Nernst-Planck model for electrodiffusion. Table 2 details the values used in this work.

\section{Reaction term}

We modeled the enzymatic reaction with Michaelis-Menten kinetics. We first define a pseudo-first-order rate constant 42

$$
\mathrm{k}=k_{c} \mathrm{c}_{\Sigma} / \mathrm{K}_{\mathrm{M}}
$$

where $k_{c}$ is the reaction (turnover) rate, $\mathrm{c}_{\Sigma}$ is the effective concentration in the voxel that corresponds to the active site, defined as

$$
c_{\Sigma}=\frac{1}{N_{A} \pi(0.5 \Delta r)^{2} \Delta z}
$$

and $\mathrm{K}_{\mathrm{M}}$ is the Michaelis constant. Table 3 summarizes the values of the constants. The reaction term is defined by

$$
R_{i}(\mathbf{r}, t)= \begin{cases}\frac{\mathrm{k} c_{i}(\mathbf{r}, t)}{1+\frac{c_{i}(\mathbf{r}, t)}{\mathrm{K}_{\mathrm{M}}}} \delta\left(\mathbf{r}_{\mathrm{rec}}\right), & i=\mathrm{NH}_{4}^{+} \\ 0, & \text { otherwise }\end{cases}
$$

where $c_{i=\mathrm{NH}_{4}^{+}}$is the concentration of the substrate, $\delta(\mathbf{r})$ is a delta function of space and $\mathbf{r}_{\text {rec }}$ is the position of the active site in cylindrical coordinates. Adding the reaction term to Equation (3), we obtain

$$
\frac{\partial c_{i}}{\partial t}(\mathbf{r}, t)=-\nabla \cdot J_{i}(\mathbf{r}, t)-R_{i}(\mathbf{r}, t)
$$

Note that the reaction terms acts on $\mathrm{NH}_{4}^{+}$only.

\section{D numerical implementation in cylindrical coordinates}

Space discretization We discretized the simulation domain using a cylindrical coordinate system with grid cell dimensions in $r$ and $z$ equal to $\Delta r=\Delta z=0.1 \mathrm{~nm}$. These dimensions are nearly an order of magnitude smaller than the Debye length, ensuring that boundary effects near the charged surface are adequately resolved. We don't explicitly define the angular grid size $\Delta \phi$ as the system is rotationally symmetric. Owing to the symmetry of the model, we only simulated a wedge of the cylindrical simulation domain (Figure 1(D)). We placed the active site, i.e., the $\mathrm{NH}_{4}^{+}$ion sink defined in (7) at position $(r=0, z=30 \mathrm{~nm})$.

Update equations The PNP model in cylindrical coordinates is given by Equations (1), (2) and 
Table 3: Definitions of variables and default values

\begin{tabular}{ccccc} 
Variable & definition & value & unit & ref. \\
\hline$k_{c}$ & catalytic rate & 10 & $1 / \mathrm{s}$ & $\underline{30}$ \\
$c_{\Sigma}$ & effective concentration at sink & $2.12 \times 10^{6}$ & $\mathrm{M}$ & \\
$\mathrm{K}_{\mathrm{M}}$ & Michaelis constant & 133 & $\mathrm{nM}$ & 43
\end{tabular}

$$
\begin{aligned}
\frac{\partial c_{i}}{\partial t}(r, \phi, z, t) & = \\
& -\left[\frac{1}{r} \frac{\partial\left(r J_{r, i}\right)}{\partial r}(r, \phi, z, t)\right. \\
& +\frac{1}{r} \frac{\partial J_{\phi, i}}{\partial \phi}(r, \phi, z, t) \\
& \left.+\frac{\partial J_{z, i}}{\partial z}(r, \phi, z, t)\right]-R_{i}(r, \phi, z, t) .
\end{aligned}
$$

The angular components $E_{\phi, i}$ and $J_{\phi, i}$ vanish everywhere as the system is rotationally symmetric. The discretized equations are given by:

$$
\begin{aligned}
& E_{r, i}\left(u+\frac{1}{2}, v, w\right)^{(t+1)}= \\
& E_{r, i}\left(u+\frac{1}{2}, v, w\right)^{(t)}-\Delta t \sum_{i=1}^{m} \frac{\mathrm{F} z_{i}}{\epsilon} J_{r, i}\left(u+\frac{1}{2}, v, w\right)^{(t)} \\
& E_{z, i}\left(u, v, w-\frac{1}{2}\right)^{(t+1)}= \\
& E_{z, i}\left(u, v, w-\frac{1}{2}\right)^{(t)}-\Delta t \sum_{i=1}^{m} \frac{\mathrm{F} z_{i}}{\epsilon} J_{z, i}\left(u, v, w-\frac{1}{2}\right)^{(t)}
\end{aligned}
$$

$$
\begin{aligned}
& J_{r, i}\left(u+\frac{1}{2}, v, w\right)^{(t+1)}= \\
& -D_{i} \frac{\partial c_{i}^{(t)}}{\partial r}+\frac{\mathrm{F}}{\mathrm{RT}} D_{i} z_{i} c_{i}^{(t)} E_{r, i}\left(u+\frac{1}{2}, v, w\right)^{(t+1)} \\
& J_{z, i}\left(u, v, w-\frac{1}{2}\right)^{(t+1)}= \\
& -D_{i} \frac{\partial c_{i}^{(t)}}{\partial z}+\frac{\mathrm{F}}{\mathrm{R} T} D_{i} z_{i} c_{i}^{(t)} E_{z, i}\left(u, v, w-\frac{1}{2}\right)^{(t+1)}
\end{aligned}
$$

and

$c_{i}(u, v, w)^{(t+1)}=c_{i}(u, v, w)^{(t)}-$

$\Delta t\left[\frac{\partial}{\partial r} J_{r, i}^{(t+1)}+\frac{1}{r} J_{r, i}^{(t+1)}+\frac{\partial}{\partial z} J_{z, i}^{(t+1)}+R_{i}(u, v, w)^{(t)}\right]$.

Furthermore, $\lim _{r \downarrow 0} 1 / r=\infty$ and by continuity of flux at $r=0, \lim _{r \downarrow 0} J_{r, i}=0$. By l'Hopital's rule $\lim _{r \rightarrow 0} \frac{1}{r} J_{r, i}=\frac{\partial J_{r, i}}{\partial r}$, and therefore a modified update equation at $r=0$ reads

$$
\begin{aligned}
& c_{i}(0, v, w)^{(t+1)}=c_{i}(0, v, w)^{(t)}- \\
& \Delta t\left[2 \frac{\partial}{\partial r} J_{r, i}^{(t+1)}+\frac{\partial}{\partial z} J_{z, i}^{(t+1)}+R_{i}(0, v, w)^{(t)}\right] .
\end{aligned}
$$

The concentration profiles are defined at grid points $(u, v, w)$ whereas the $J_{r, i}$ are at the middle of two consecutive $u$ points, namely $(u+$ $\left.\frac{1}{2}, v, w\right)$ and $J_{z, i}$ 's are located at $\left(u, v, w-\frac{1}{2}\right)$.

Boundary and initial conditions Starting profiles for the concentrations and electric field were calculated analytically from equilibrium solutions to the Poisson-Boltzmann equations in the absence of a nanochannel. ${ }^{[30}$ We assumed no current or ion flux at $t=0$. We applied a diffusive boundary condition (BC) to the top domain boundary $(z=0)$ to allow ion influx or efflux from the simulation domain, i.e. the flux at the top domain boundary is determined by

$$
J_{z, i}\left(u, v,-\frac{1}{2}\right)=-\frac{\Delta t}{\Delta z} D_{i}\left[c_{i}(u, v, 0)-c_{i, \text { bulk }}\right]
$$

and Neumann BCs of $\partial c / \partial u=0$, i.e., 'wall' conditions, to the bottom domain $(z=40 \mathrm{~nm})$ and the S-layer boundaries to prevent diffusion. The remaining boundaries are periodic.

We calculated the Debye length $l_{\mathrm{D}}=\kappa^{-1}$ and the Gouy-Chapman length $l_{\mathrm{GC}}$ at equilibrium given a surface charge density $\rho=0.02 \mathrm{e}^{-} \AA^{-2}$ and a bulk $\mathrm{NaCl}$ concentration $c_{\mathrm{NaCl} \pm \text {,bulk }}$ of $0.14 \mathrm{M}$ as $l_{\mathrm{D}}=\left[\epsilon k_{B} T /\left(8 \pi \mathrm{e}^{2} n_{0}\right)\right]^{1 / 2}=8.15 \AA$, and $l_{\mathrm{GC}}=\epsilon T /(2 \pi e|\sigma|)=1.14 \AA$, where $k_{B}$ denotes the Boltzmann constant, and $c_{0}$ is the bulk concentration in moles per $\mathrm{m}^{3}$.

\section{Simulations}

The numerical model was evolved in time by a Poisson-Nernst-Planck equation solver written 

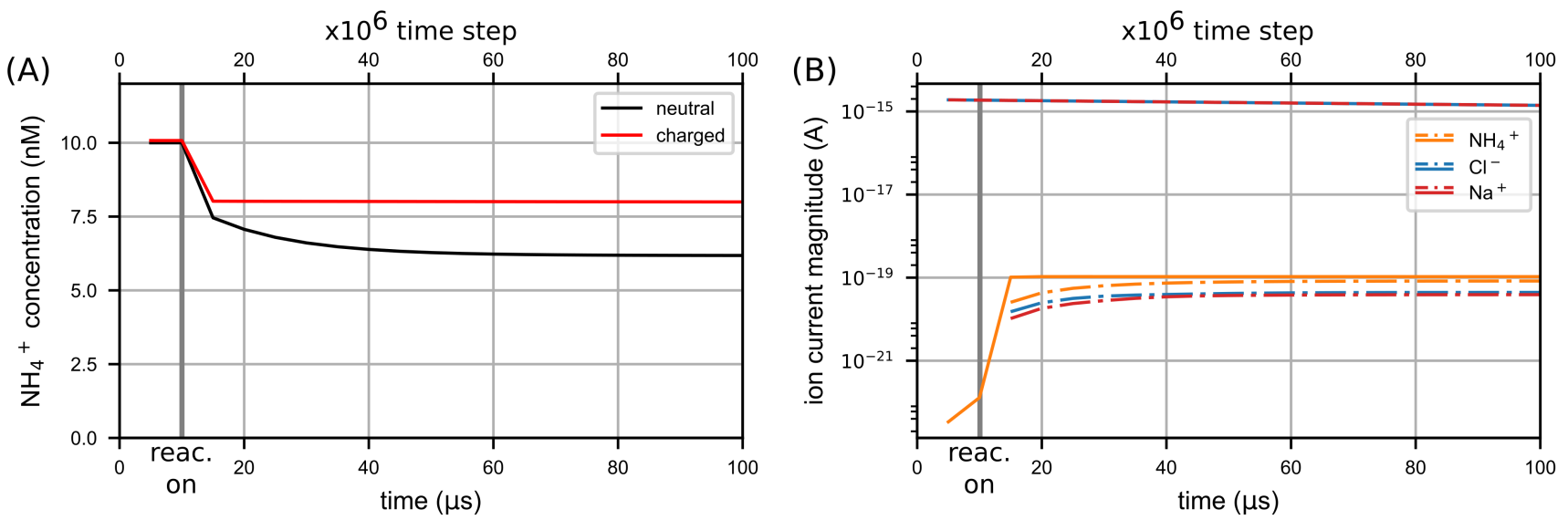

Figure 9: (A) $\mathrm{NH}_{4}^{+}$concentration at the active site vs. simulation time. Time step size is $1 \times 10^{-12} \mathrm{~s}$. (B) Current of each ion in the nanochannel vs. simulation time in logarithmic scale. Dash-dot (solid) lines represent neutral (charged) S-layer. The reaction is turned on at $t=10 \mu \mathrm{s}$.

in $\mathrm{C}++$. Each simulation was equilibrated for $10 \mu s$ with the reaction term switched off. We monitored ion concentrations at the sink and ion fluxes over the nanochannel to ensure the system had reached equilibrium (Figure 9). We then switched on the reaction term, and simulated the system for another $80 \mu \mathrm{s}$. The time step size $\Delta t=1 \times 10^{-12} \mathrm{~s}$ was selected to ensure numerical stability while maximizing simulation speed.

\section{Numerical convergence}

To determine if the simulations had converged to steady state, we monitored the concentration of $\mathrm{NH}_{4}^{+}$at the active site in the simulation with a neutral S-layer and a charged S-layer. Simulations with a charged S-layer converged within $5 \mu$ s after switching on the reaction (Figure $9(\mathrm{~A}))$. By contrast, the neutral S-layer simulations reached a steady state only $50 \mu$ s after switching on the reaction. Slow convergence in the presence of an uncharged membrane is generally attributed to slow co-diffusion of oppositely charged ion species. $\underline{44}$

\section{Electrical properties}

We calculated potentials across the pore by integrating $\mathbf{E}_{z}$ with respect to $z$ across the pore from the upper reservoir to the sink $(z=$ $[10.0,30.0] \mathrm{nm})$ and averaging over the area of the pore $(r=[0,7) \mathrm{nm})$. The chemi- cal potential for ion species $i$ is calculated as $E_{c, i}=k_{B} T \ln \left(c_{i} / c_{B i}\right)$, where $c_{B i}$ is the bulk concentration of species $i$; the electrical potential is calculated as $E_{e, i}=z_{i} \mathrm{eV}$. The electrical current for ion species $i$ is calculated by $I_{i}=2 z_{i} N_{A} \mathrm{e} \Delta r \pi \sum_{r}\left(J_{i, \text { avg }}(r, z) r_{i}\right)$, where $J_{i, \text { avg }}(r, z)$ is obtained by averaging $J_{i}(r, z)$ over the depth of the pore $([17.8,22.4) \mathrm{nm})$ and $r_{i}$ is the distance of the $i$-th voxel from the $r=0$ axis. The sign of $G=I / V$ follows the convention that flux of negative charge toward positive potential $\mathrm{V}$ is positive conductance.

\section{Acknowledgements}

JH was supported in part by the National Science Foundation Graduate Research Fellowship Program (NSF-GRFP) and the US Department of Energy Office of Science Graduate Student Research Program (DOE-SCGSR). HvdB is supported by NIH GM123159. The authors gratefully acknowledge Christopher A. Francis and Bradley B. Tolar for providing N. limnia strain SFB1 cells, and A. Majumdar for stimulating discussions. Simulations were partly performed at the Stanford Research Computing Center and the Linac Coherent Light Source (LCLS).

Supporting Information Available: A table comparing ion currents observed from a $2 \mathrm{D}$ approximation of our $3 \mathrm{D}$ model with a true $2 \mathrm{D}$ model. A figure of the ion flux profiles in the 
nanochannel, and a figure of the $\mathrm{NH}_{4}^{+}$flux in PPS. This material is available free of charge via the Internet at http://pubs.acs .org.

\section{References}

1. Roux B. Ion channels and ion selectivity Essays Biochem 2017, 61, 201-209.

2. Tagliazucchi, M.; Szleifer, I. Transport mechanisms in nanochannels and nanochannels: can we mimic nature? Mater. Today 2015, 18, 1369.

3. Pérez-Mitta, G; Albesa, A.G.; Trautmann, C; Toimil-Molares, M.-E.; Azzaroni, O. Bioinspired integrated nanosystems based on solid-state nanochannels: 'iontronic' transduction of biological, chemical and physical stimuli. Chem. Sci. 2017, 8, 890913.

4. Feng, J.; Graf, M.; Liu, K.; Ovchinnikov, D.; Dumcenco, D.; Heiranian, M; Nandigana, V.; Aluru, N. R. Kis, A.; Radenovic, A. Single-layer MoS2 nanochannels as nanopower generators. Nature 2016, 536, 197-200.

5. Sui, X.; Zhang, Z.; Li, C.; Gao, L.; Zhao, Y.; Yang, L.; Wen, L.; Jiang, L. Engineered Nanochannel Membranes with Diode-like Behavior for Energy Conversion over a Wide pH Range. ACS Appl. Mater. Interfaces $\mathbf{2 0 1 9}$

6. Jain, M.; Olsen, H.E.; Paten, B.; Akeson, M.; The Oxford nanochannel MinION: delivery of nanochannel sequencing to the genomics community. Genome Biol. 2016, $17,239$.

7. Haynes, T.; Smith, I.P.S.; Wallace, E.J.; Trick, J.L.; Sansom, M.S.P.; Khalid, S. Electric-Field-Driven Translocation of ssDNA through Hydrophobic Nanopores. ACS Nano 2018, 12, 8208-8213.

8. Pan, Y.; Zhou, Y.; Zhao, Q.; Dou, Y.; Chou, S.; Cheng, F.; Chen, J.; Liu,
H.K.; Jiang, L.; Dou, S.X. Introducing ion-transport-regulating nanochannels to lithium-sulfur batteries. Nano Energy 2017, 33, 205.

9. Shen, L.; Wu. H.B.; Liu, F.; Brosmer J.L.; Shen, G., Wang, X.; Zink, J.I.; Xiao, Q.; Cai, M.; Wang, G.; Lu, Y.; Dunn, B. Creating Lithium-Ion Electrolytes with Biomimetic Ionic Channels in Metal-Organic Frameworks. Adv. Mater., 2018, 30, 1707476.

10. Siria, A.B.; Bocquet, M.-L.; Bocquet, L. New avenues for the large-scale harvesting of blue energy. Nat. Rev. Chem. 2017, 1, 0091.

11. Daiguji, H.; Yang, P.; Majumdar, A. Ion Transport in Nanofluidic Channels. Nano Lett. 2004, 7, 137-142.

12. Stein, D.; Kruithof, M.; Dekker, C. Surfacecharge-governed ion transport in nanofluidic channels Phys. Rev. Lett. 2004, 93, 035901.

13. Kim, S.J.; Wang, Y.-C.; Lee, J.H.; Jang, H.; Han, J. Concentration Polarization and Nonlinear Electrokinetic Flow near a Nanofluidic Channel. Phys. Rev. Lett. 2007, 99, 044501.

14. Karnik, R.; Duan, C.; Castelino, K.; Daiguji, D.; Majumdar, A. Rectification of Ionic Current in a Nanofluidic Diode. Nano Lett. 2007, 7, 547-551.

15. Zangle, T.A.; Mani, A.; Santiago, J.G.; Theory and experiments of concentration polarization and ion focusing at microchannel and nanochannel interfaces. Chem. Soc. Rev. 2010, 39, 1014-1035.

16. Bruno, G.; Di Trani, N.; Lyle Hood, R.; Zabre, E.; Filgueira, C.S.; Canavese, G.; Jain, P.; Smith, Z.; Demarchi, D.; Hosali, S.; Pimpinelli, A.; Ferrari, M.; Grattoni, A. Unexpected behaviors in molecular transport through size-controlled nanochannels down to the ultra-nanoscale. Nature Commun. 2018, 8, 1682. 
17. Jubin, L.; Poggioli, A.; Siria, A.; Bocquet, L. Dramatic pressure-sensitive ion conduction in conical nanochannels. Proc. Natl. Acad. Sci. USA 2018, 115, 4063-4068.

18. Gorman-Lewis, D.; Martens-Habbena, W.; Stahl, D.A. Thermodynamic characterization of proton-ionizable functional groups on the cell surfaces of ammonia-oxidizing bacteria and archaea. Geobiology 2014, 12, 157-171.

19. Stahl, D.A.; de la Torre, J.R. Physiology and Diversity of Ammonia-Oxidizing Archaea. Annu. Rev. Microbiol. 2012, 66, 83.

20. Mosier, A.C.; Lund, M.B.; Francis, C.A. Ecophysiology of an Ammonia-Oxidizing Archaeon Adapted to Low-Salinity Habitats. Microb. Ecol. 2012, 64, 955.

21. Leininger, S.; Urich, T.; Schloter, M.; Schwark, L.; Qi, J.; Nicol, G.W.; Prosser, J.I.; Schuster, S.C.; Schleper, C. Archaea predominate among ammoniaoxidizing prokaryotes in soils. Nature 2006, 442, 806-809.

22. Wuchter, C.; Abbas, B.; Coolen, M.J.L.; Herfort, L.; van Bleijswijk, J.; Timmers, P.; Strous, M.; Teira, E.; Herndl, G.J.; Middelburg, J.J.; Schouten, S.; Sinninghe Damsté, J.S.; Archaeal nitrification in the ocean. Proc Natl Acad Sci USA 2006, 33, 12317-12322.

23. Tolar, B.B.; Herrmann, J.; Bargar, J.R.; van den Bedem, H.; Wakatsuki, S,; Francis, C.A. Integrated structural biology and molecular ecology of N-cycling enzymes from Ammonia-Oxidizing Archaea. . Environ. Microbiol. Rep. 2017, 9, 484-491.

24. Fagan, R.P.; Fairweather, N.F. Biogenesis and functions of bacterial S-layers. Nat. Rev. Microbiol. 2014 211-222.

25. Arbing, M.A.; Chan, S.; Shin, A.; Phan, T.; Ahn, C.J.; Rohlin, L.; Gunsalus, R.P. Structure of the surface layer of the methanogenic archaean Methanosarcina acetivorans. Proc. Natl. Acad. Sci. USA 2012, 109, 11812.

26. Bharat, T.A.M.; Kureisaite-Ciziene, D.; Hardy, G.G.; Yu, E.W.; Devant, J.M.; Hagen, W.J.H.; Brun, Y.V.; Briggs, J.A.G; Łöwe J. Structure of the hexagonal surface layer on Caulobacter crescentus cells. $\mathrm{Na}$ ture Microbiol. 2017, 2, 17059.

27. Sleytr, U.B.; Schuster, B.; Egelseer, E.-M.; Pum, D. S-layers: principles and applications. FEMS Microbiol. Rev. 2014, 38 823864.

28. Sára, M.; Sleytr, U.B. Molecular Sieving through S Layers of Bacillus stearothermophilus Strains. J. Bacteriol. 1987, 169, 4092-4098.

29. Ho, C.; Qiao, R.; Heng, J.B.; Chatterjee, A.; Timp, R.J.; Aluru, N.R.; Tim, G. Electrolytic transport through a synthetic nanometer-diameter pore. Proc Natl Acad Sci USA 2005, 102, 10445-10450.

30. Li, P.-N.; Herrmann, J.; Tolar, B.B.; Poitevin, F.; Ramdasi, R.; Bargar, J.R.; Stahl, D.A.; Jensen, G.J.; Francis, C.A.; Wakatsuki, S.; van den Bedem, H. Nutrient transport suggests an evolutionary basis for charged archaeal surface layer proteins. ISME J. 2018, 12, 2389-2402.

31. Mosier, A.C.; Lund, M.B.; Francis, C.A. Ecophysiology of an Ammonia-Oxidizing Archaeon Adapted to Low-Salinity Habitats. Microb. Ecol. 2012, 64, 955.

32. Singh K.P.; Guo, C. Current-voltage characteristics influenced by the nanochannel diameter and surface charge density in a fluidic field-effect-transistor. Phys. Chem. Chem. Phys. 2017, 19, 15701.

33. Fan, R.; Yue, M.; Karnik, R.; Majumdar, A.; Yang, P. Polarity Switching and Transient Responses in Single Nanotube Nanofluidic Transistors. Phys. Rev. Lett. 2005, 086607. 
34. Herrmann, J.; Jabbarpour, F.; Bargar, P.G.; Nomellini, J.F.; Li, P.-N.; Lane, T.J.; Weiss, T.M.; Smit, J.; Shapiro, L. Wakatsuki, S. Environmental Calcium Controls Alternate Physical States of the Caulobacter Surface Layer. Biophys. J. 2017, 112, 1841.

35. Qin, W.; Heal, K.R.; Ramdasi, R.; Kobelt, J.N.; Martens-Habbena, W.; Bertagnolli, A.D.; Amin, S.A.; Walker, C.B.; Urakawa, H.; Könneke, M.; Devol, A.H.; Moffett, J.W.; Armbrust, E.V.; Jensen, G.J.; Ingalls, A.E.; Stahl, D.A. Nitrosopumilus maritimus gen. nov., sp. nov., $N i$ trosopumilus cobalaminigenes sp. nov., $\mathrm{Ni}$ trosopumilus oxyclinae sp. nov., and . $\mathrm{Ni}$ trosopumilus ureiphilus sp. nov., four marine ammonia-oxidizing archaea of the phylum Thaumarchaeota. Int. J. Syst. Evol. Microbiol. 2017, 67, 5067-5079.

36. Karnik, R.; Fan, R.; Yue, M.; Li, D.; Yang, P.; Majumdar, A. Electrostatic Control of Ions and Molecules in Nanofluidic Transistors. Nano Lett. 2005, 5, 943-948.

37. Nandigana, V.V.R.; Aluru N.R. Understanding anomalous current-voltage characteristics in microchannel-nanochannel interconnect devices. J. Colloid Interface Sci. 2012, 15, 162-171.

38. Yossifon, G; Mushenheim, P.; Chang, Y.C.; Chang, H.-C. Nonlinear current-voltage characteristics of nanochannels. Phys. Rev. E, 2009, 79, 046305.

39. Constantin, D.; Siwy, Z.S. Poisson-NernstPlanck model of ion current rectification through a nanofluidic diode. Phys. Rev. E 2007, 76, 041202.

40. Cohen, H.; Cooley, J. The Numerical Solution of the Time Dependent Nernst-Planck equations. Biophys. J., 1965, 5, 145-162.

41. Jasielec, J.J. Computer Simulations of Electrodiffusion Problems Based on NernstâĂŞPlanck and Poisson Equations. Comput. Mater. Sci. 2012, 63, 75 (2012).
42. Lyons, M.E.G.; Greer, J.C.; Fitzgerald, C.A.; Bannon, T.; Barlett, P.N. ReactionDiffusion with Michaelis-Menten Kinetics in Electroactive Polymer Films. Part 1. The Steady-State Amperometric Response. Analyst 1996, 121, 715-731.

43. Martens-Habbena, W.; Berube, P.M.; Urakawa, H.; de la Torre, J.R.; Stahl, D.A. Ammonia oxidation kinetics determine niche separation of nitrifying Archaea and Bacteria, Nature 2009, 461, 976-981.

44. van Oeffelen, L.; van Roy, W.; Idrissi, H.; Charlier, D.; Lagae, L.; Borghs, G. Ion Current Rectification, Limiting and Overlimiting Conductances in nanochannels. PLoS ONE 2015, 10, e0124171.

45. Mitscha-Baude, G.; Buttinger-Kreuzhuber, A.; Tulzer, G.; Heitzinger, C. Adaptive and Iterative Methods for Simulations of Nanopores with the PNP-Stokes Equations. J. Comput. Phys. 2017, 338 452-476.

46. Chaudhry, J.; Comer, J.; Aksimentiev, A.; Olson, L. A Stabilized Finite Element Method for Modified Poisson-NernstPlanck Equations to Determine Ion Flow Through a Nanochannel. Commun. Comput. Phys., 2014, 15, 93-125 (2014)

47. Gamble, T.; Decker, K.; Plett, T.S.; Pevarnik, M.; Pietschmann, J.-F.; Vlassiouk, I.; Aksimentiev, A.; Siwy, Z.S. Rectification of Ion Current in Nanochannels Depends on the Type of Monovalent Cations: Experiments and Modeling. J Phys Chem C 2014, 118, 9809-9819.

48. Siwy, Z. Ion-Current Rectification in nanochannels and Nanotubes with Broken Symmetry. Adv. Funct. Mater. 2006, 16, 735-746.

49. Karnik, R.; Duan, C.; Castelino, K.; Daiguji, H.; Majumdar, A. Rectification of Ionic Current in a Nanofluidic Diode. Nano Lett. 2007, 7, 547-551. 
Transport_Properties_Of_nanoporous_Chemically-Forced... (4.19 MiB) view on ChemRxiv • download file 


\section{Supplemental Information to:}

\section{Transport properties of nanoporous, chemically forced biological lattices.}

Po-Nan Li, Jonathan Herrmann, Soichi Wakatsuki, and Henry van den Bedem

Table S1: Ion currents in the charged case (surface charge density $\rho=0.02 \mathrm{e}^{-} \AA^{-2}$ ) observed using a $2 \mathrm{D}$ approximation of the $3 \mathrm{D}$ model in this manuscript vs. the $2 \mathrm{D}$ model ${ }^{[30}$

2D approximation with 3D model

\begin{tabular}{rrrr}
\hline \hline Species & $\mathrm{NH}_{4}^{+}$ & $\mathrm{Cl}^{-}$ & $\mathrm{Na}^{+}$ \\
\hline Current & $1.04 \mathrm{E}-18 \mathrm{~A}$ & $1.57 \mathrm{E}-20 \mathrm{~A}$ & $-1.02 \mathrm{E}-18 \mathrm{~A}$ \\
& & \\
& $\mathrm{D}$ model & \\
\hline Species & $\mathrm{NH}_{4}^{+}$ & $\mathrm{Cl}^{-}$ & $\mathrm{Na}^{+}$ \\
\hline Current & $3.30 \mathrm{E}-20 \mathrm{~A}$ & $1.60 \mathrm{E}-20 \mathrm{~A}$ & $-1.60 \mathrm{E}-20 \mathrm{~A}$
\end{tabular}



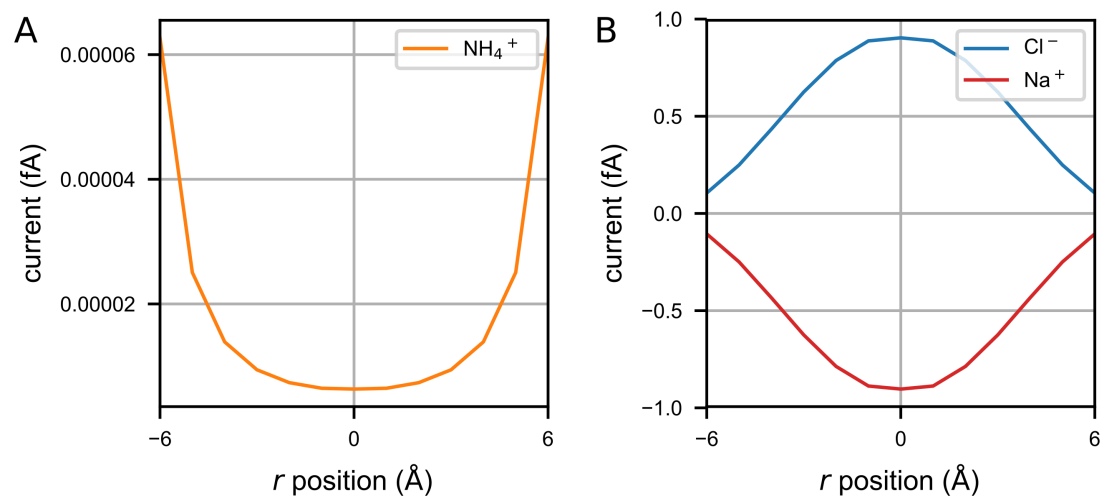

Figure S1: Current profiles in a charged nanochannel with surface charge density $0.02 \mathrm{e}^{-} \AA^{-2}$ 


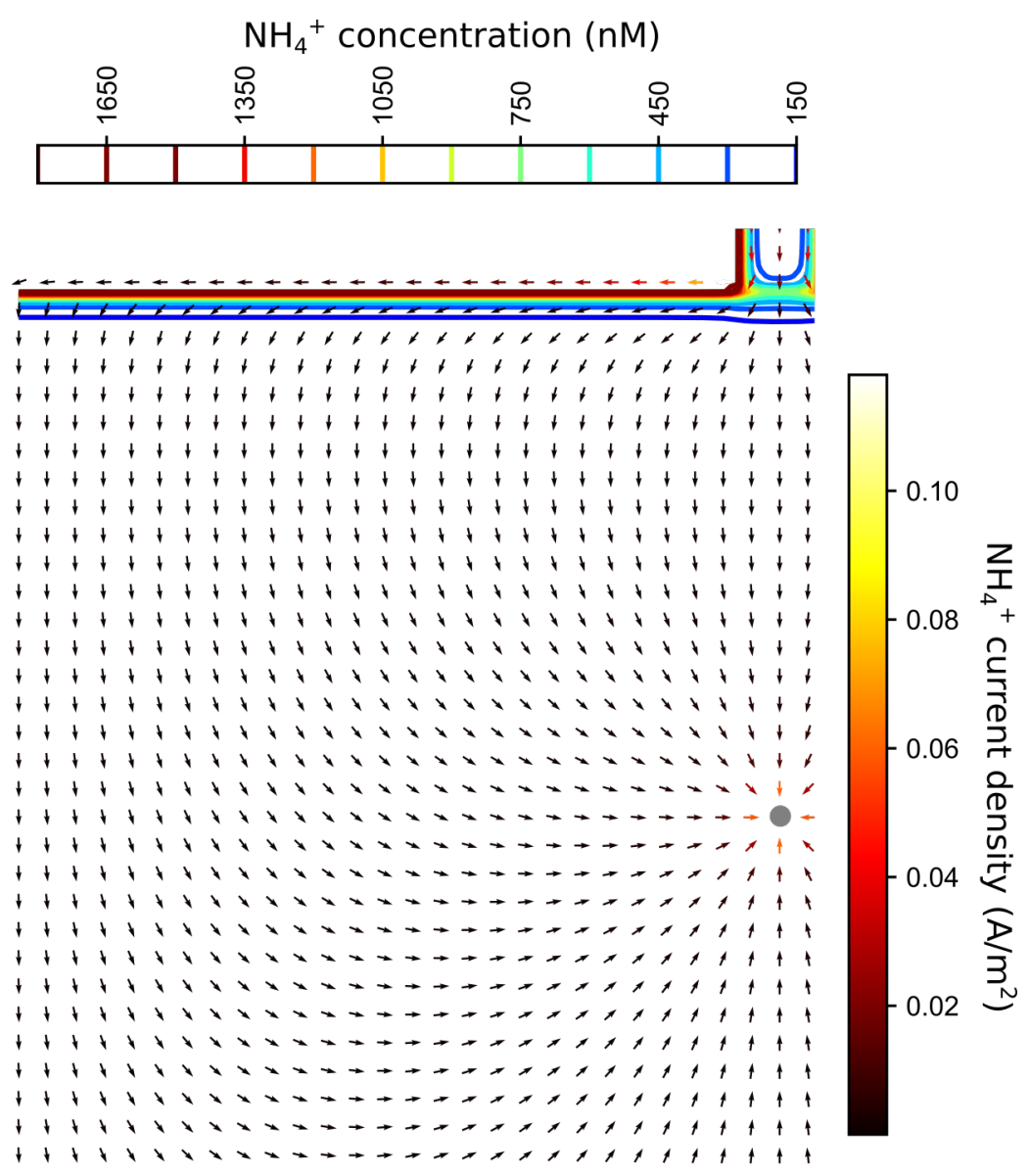

Figure S2: Quiver plot showing the direction of $\mathrm{NH}_{4}^{+}$fluxes near the active site, marked as a gray circle. The contour lines show the concentration gradient of $\mathrm{NH}_{4}^{+}$near the surface layer. 
